\title{
Vibration Annihilation of Sandwiched Beam with MROF DTSMC
}

\author{
Vivek Rathi, Ahmad Ali Khan \\ Mechanical Engineering Department, AMU, Aligarh, India \\ Email: logonrathi@yahoo.co.in, khanaali@rediffmail.com
}

How to cite this paper: Rathi, V. and Khan, A.A. (2017) Vibration Annihilation of Sandwiched Beam with MROF DTSMC. Engineering, 9, 755-778.

https://doi.org/10.4236/eng.2017.99046

Received: August 24, 2017

Accepted: September 18, 2017

Published: September 21, 2017

Copyright $\odot 2017$ by authors and Scientific Research Publishing Inc. This work is licensed under the Creative Commons Attribution International License (CC BY 4.0).

http://creativecommons.org/licenses/by/4.0/

\begin{abstract}
In the present paper, an analytical model of a flexible beam fixed at an end with embedded shear sensors and actuators is developed. The smart cantilever beam model is evolved using a piezoelectric sandwich beam element, which accommodates sensor and actuator embedded at distinct locations and a regular sandwiched beam element, having rigid foam at the core. A FE model of a piezoelectric sandwich beam is evolved using laminated beam theory in MATLAB $^{\circledast}$. Each layer behaves as a Timoshenko beam and the cross-section of the beam remains plane and rotates about the neutral axis of the beam, but it does not remain normal to the deformed longitudinal axis. Keeping the sensor and actuator location fixed in a MIMO system, state space models of the smart cantilever beam is obtained. The proper selection of control strategy is very crucial in order to obtain the better control. In this paper a DSM controller designed to control the first three modes of vibration of the smart cantilever beam and their performances are represented on the basis of control signal input, sensor output and sliding functions. It is found that DSM controller provides superior control than other conventional controllers and also MROF DSM controller is much better than SISO DSM controller.
\end{abstract}

\section{Keywords}

Active Vibration Control, Finite Element, LTI, DSMC, DQSMC, MATLAB, MIMO

\section{Introduction}

Smart structures [1] are systems having particular functions viz. sensing, processing, actuation and making them suitable for structural health conditioning, vibration suppression of structures. Piezoelectric materials are found most suitable to be used as active components in smart structures [2]. The apposite- 
ness of piezoelectric materials as sensors and actuators has gained the focus in health monitoring of structures like beams, plates, and shells [3]-[15]. Krommer [16], Rao and Sunar [17] have shown the implementation of piezoelectric materials as both for sensing and actuation. Active control through bonded piezo components was studied by Moita et al. [18]. An optimal linear quadratic generator control strategy to control the structures is advised by Ulrich et al. Young et al. [20] presented a finite element simulation of flexible structures with output feedback controllers. Aldraihem et al. [21] developed the model of the laminated beam based on EBT and TBT. Abramovich [22] has obtained an analytical formulation and closed form results of the laminated beam based on TBT with piezoelectric sensors and actuators. Chandrashekhara and Vardarajan [23] acquainted a finite element model of the laminated beam to evolve deflection in beams with various end conditions. Sun and Zhang [24] have suggested the basis of shear mode to produce transverse deflection in embedded structures. Aldraihem and Khdeir [25] expounded the analytical model and exact solution of Timoshenko beam with shear and extensional piezoelectric actuators. Zhang and Sun [26] have presented an analytical model of surface mounted beam with shear piezo actuators at the core. The top and bottom layers obey EBT and core obeys TBT. Donthireddy and Chandrashekhara [27] have proposed a model with embedded piezoelectric components. Rathi and Khan [28] have modeled a smart cantilever beam with surface mounted and embedded shear sensors and actuators on the basis of TBT and justify that embedded components of flexible structures provide better control than surface mounted arrangement and also emphasized on optimal location of sensors and actuators in embedded beam.

Chammas and Leondes [29] [30] have presented the pole assignment by piecewise constant output feedback for LTI systems while Werner and Furuta [31] [32] focussed on fast output sampling for LTI system. Janardhan et al. [33] designed a controller based on MROF using the samples of control input and sensor output at different sampling rates. Bandyopadhyay et al. [34] adduced a DTSM control that has the use of switching function in control results in QSMC.

A numerous types of control policies for the SISO and MIMO state space presentation of the active structures using the Multiple Rate Output Feedback (MROF) dependent Discrete Sliding Mode Control (DSMC) approach is depicted in this monograph. The key objective instigating this control technique is to constrain and damp out the flexural or transverse vibrations of active beam when they are subjected to external annoyance. The control technique used on the basis of Bartoszewicz law and does not need to use switching in control function and hence eradicate chattering. This method does not need the reconnaissance of the system states for feedback being using solely the output samples for designing the controller. The schematic espousal is more viable and may be easy to accomplish in true life applications.

\section{Discrete Time Sliding Mode Control (DTSMC)}

Bartoszewicz [35] adduce a quasi-sliding mode control (QSMC) technique with- 
out using a switching function in control and has the property of finite time convergence to the QSM band. A discrete output feedback sliding mode control algorithm in [33] based Bartoszewicz's control law [35] and MROF [36] is used for the vibration suppression of flexible structures.

In the present situation, the disturbance is the external force input $r(t)$ in form of impulsive force applied to the free end of the beam and hence producing the vibration. DSM controllers with multirate output feedback plan evolved and applied to the system with the plant to attenuate the vibrations earliest. The methodology is described as follows:

Consider a CT SISO system sampled with an interval $\alpha$ seconds and given as

$$
\begin{gathered}
x(n+1)=\Theta_{\alpha} x(n)+\Delta \Theta_{\alpha} x(n)+\Upsilon_{\alpha} u(n)+f(n) \\
y(n)=C x(n)
\end{gathered}
$$

where, $\Delta \Theta_{\alpha}$ is the uncertainty in the state, $f(n)$ is an external disturbance vector and $\left(\Theta_{\alpha}, \Upsilon_{\alpha}\right)$ being controllable and $\left(\Theta_{\alpha}, C\right)$ being observable. Let us choose the disturbance vector as

$$
\tilde{\zeta}(n)=\Delta \Theta_{\alpha} x(n)+f(n)
$$

Let the desired sliding manifold be governed by the parameter vector $p^{T}$ such that $p^{\mathrm{T}} \Upsilon_{\alpha} \neq 0$ and resulting quasi-sliding motion is stable and assume that disturbance be bounded such that

$$
\zeta(n)=p^{\mathrm{T}} \tilde{\zeta}(n)
$$

Which satisfies the inequality

$$
\zeta_{-1} \leq \zeta(n) \leq \zeta_{+1}
$$

where, $\zeta_{-1}$ and $\zeta_{+1}$ are lower and upper bounds on the disturbance. We take,

$$
\zeta_{0}=0.5\left(\zeta_{+1}+\zeta_{-1}\right) \text { and } \delta_{\zeta}=0.5\left(\zeta_{+1}-\zeta_{-1}\right)
$$

The switching surface is given by

$$
S(n)=p^{\mathrm{T}} x(n)
$$

The QS mode is the motion such that $|S(n)| \leq \eta$, where the positive constant $\eta$ is termed as quasi-sliding mode bandwidth. A significant reduction of control effort and better quality of quasi-sliding mode control is found. A reaching law advised by Bartoszewicz [35] is as follows

$$
S(n+1)=\zeta(n)-\zeta_{0}+S_{\zeta}(n+1)
$$

where, $\zeta(n)$ is given from Equation (4) and $S_{\zeta}(n)$ is a known function that satisfies the following two states, when

1) If $|S(0)|>2 \delta_{\zeta}$, then $S_{\zeta}(0)=S(0), S_{\zeta}(n) S_{\zeta}(0) \geq 0$, for all $n \geq 0$

2) If $|S(0)|<2 \delta_{\zeta}$, then $S_{\zeta}(0)=0$, for all $n \geq 0$

The value of the positive integer $n^{*}$ is chosen by Engineer so as to have a compromise in between rapid convergence an amplitude of control input $u(t)$. By controlling the decay rate $\left(n^{*}\right)$, the convergence of $S(n)=0$ acclimated 
with the reaching law and the two conditions of the function $S_{\zeta}(n)$ implies that the reaching law condition is satisfied and for all $n \geq n^{*}$, the QS mode in the $\delta_{\zeta}$ vicinity of the sliding plane $S(n)=p^{\mathrm{T}} x(n)=0$ presented. One possible function for $S_{\zeta}(n)$, when $|S(0)|>2 \delta_{\zeta}$, can be described as

$$
\begin{gathered}
S_{\zeta}(n)=\frac{n^{*}-n}{n^{*}} S(0), n=0,1,2, \cdots, n^{*} \\
n^{*}<\frac{|S(0)|}{2 \delta_{\zeta}}
\end{gathered}
$$

The control law satisfying the reaching law (Equation (7)) and get sliding mode for the system as given in Equation (4) can be computed to be

$$
u(n)=\left(p^{\mathrm{T}} \Upsilon_{\alpha}\right)^{-1}\left\{p^{\mathrm{T}} \Theta_{\alpha} x(n)+\zeta_{0}-S_{\zeta}(n+1)\right\}
$$

When control input given in Equation (11) substituted into the system, it would sure for any $n>n^{*}$, the switching function would satisfy the expression

$$
|S(n)|=|\zeta(n-1)-\zeta(0)| \leq \delta_{\zeta}
$$

Thus, system states adjudicate within a QSM band having less than half bandwidth as given in [37]. From [33] MROF based algorithm using an advanced reaching law can be attained. Let the advanced reaching law be [35] given as

$$
S(n+1)=\zeta(n)-\zeta(0)+o(n-1)-o_{0}+S_{\zeta}(n+1)
$$

A new variable $o(n)$ is incorporated here. The control input generated can be given by using algorithm in [33],

$$
u(n)=-\left(p^{\mathrm{T}} \Upsilon_{\alpha}\right)^{-1}\left\{p^{\mathrm{T}} \Theta_{\alpha} L_{y} y_{n}+p^{\mathrm{T}} \Theta_{\alpha} L_{u} u(n-1)+\zeta_{0}+o_{0}-S_{\zeta}(n+1)\right\}
$$

Here,

$$
\begin{gathered}
L_{y}=\Theta_{\alpha} C_{0}^{-1}, L_{u}=\Upsilon_{\alpha}-C_{0}^{-1} D_{0}, L_{\zeta}=I-C_{0}^{-1} C_{\zeta} \\
C_{0}=\left[\begin{array}{c}
C \\
C \Theta \\
C \Theta^{2} \\
\vdots \\
C \Theta^{N-1}
\end{array}\right], D_{0}=\left[\begin{array}{c}
0 \\
C \Upsilon \\
C(\Theta \Upsilon+\Upsilon) \\
\vdots \\
C \sum_{k=0}^{N-2} \Theta^{k} \Upsilon
\end{array}\right], C_{\zeta}=\left[\begin{array}{c}
C\left(\sum_{k=0}^{N-1} \Theta^{k}\right)^{-1} \\
C\left(\sum_{k=0}^{1} \Theta^{k}\right)\left(\sum_{k=0}^{N-1} \Theta^{k}\right)^{-1} \\
\vdots \\
C\left(\sum_{k=0}^{N-2} \Theta^{k}\right)\left(\sum_{k=0}^{N-1} \Theta^{k}\right)^{-1}
\end{array}\right]
\end{gathered}
$$

with $o_{0}=0.5\left(o_{+1}+o_{-1}\right)$ and $\delta_{o}=0.5\left(o_{+1}-o_{-1}\right)$ are the mean and variation of the function of uncertainty. $O_{+1}$ and $O_{-1}$ are the upper and lower bounds of $o(n)$. The variable $o(n)$ represents the disturbance effect on sampled output

$$
o(n)=p^{\mathrm{T}} \Theta_{\alpha} L_{\zeta} \tilde{\zeta}_{n}
$$

The bounds on $o(n)$ are given as $o_{-1} \leq o(n) \leq O_{+1}$, the value of $N$ is cho- 
sen to be greater the observability index $j$ of the system defined as for system given by triplet $(A, B, C)$ is the minimum integer value of $j$ such that

$$
\operatorname{Rank}\left(\begin{array}{c}
C \\
C A \\
\vdots \\
C A^{j-1}
\end{array}\right)=\operatorname{Rank}\left(\begin{array}{c}
C \\
C A \\
\vdots \\
C A^{j}
\end{array}\right)
$$

Thus the control input can be estimated by using the past output samples and immediate past input. But at $n=0$, there are no past outputs for use in control, here $u(0)$ is obtained by neglecting $o(n-1)$ and $o_{0}$ (no disturbance before $n=0$ to affect the system), so we have,

$$
u(0)=-\left(p^{\mathrm{T}} \Upsilon_{\alpha}\right)^{-1}\left\{p^{\mathrm{T}} \Theta_{\alpha} x_{0}+\zeta_{0}-S_{\zeta}(1)\right\}
$$

When control input obtained from eq. (14) is used in system obeys reaching law

$$
\begin{gathered}
S(n+1)=\zeta(n)-\zeta_{0}+o(n-1)-o_{0}+S_{\zeta}(n+1) \\
S(n)=\zeta(n-1)-\zeta_{0}+o(n-2)-o_{0}+S_{\zeta}(n)
\end{gathered}
$$

When $n>\left(n^{*}, 2\right), S_{\zeta}(n)=0$ and hence,

$$
S(n)=\zeta(n-1)-\zeta_{0}+o(n-2)-o_{0}
$$

So we have,

$$
\begin{aligned}
& |S(n)|=\left|\zeta(n-1)-\zeta_{0}+o(n-2)-o_{0}\right| \\
& |S(n)| \leq\left|\zeta(n-1)-\zeta_{0}\right|+\left|o(n-2)-o_{0}\right|
\end{aligned}
$$

This can be written as

$$
|S(n)| \leq \delta_{\zeta}+\zeta_{0}
$$

It can be emphasized that this algorithm does not need the assessment of system states for the creation of control input. This control technique is used to design a multi-rate output feedback based DSM control to attenuate the transverse disturbance in a flexible structure which is modeled on the basis of Timoshenko beam theory for 3 vibratory modes.

\section{Finite Element Modeling of an Embedded Beam}

An embedded beam consists of three layers having a piezoelectric patch with the obdurate foam in between two thick steel beams shown in Figure 1. The lead zirconate titanate (PZT) layer acts as both actuator and sensor in thickness shear actuation mode. The foam and PZT together behave like a core element to obtain embedded beam model [28].

The presumption is that the mid layer is perfectly bonded to the rest of the structure and thickness of binder is neglected (hence preventing shear-lag, slip or layer delamination during vibration) resulting a strong blend between parent structure and piezoelectric patches. The binder used between the layers have been assumed no added mass or stiffness to sensor or actuator. 


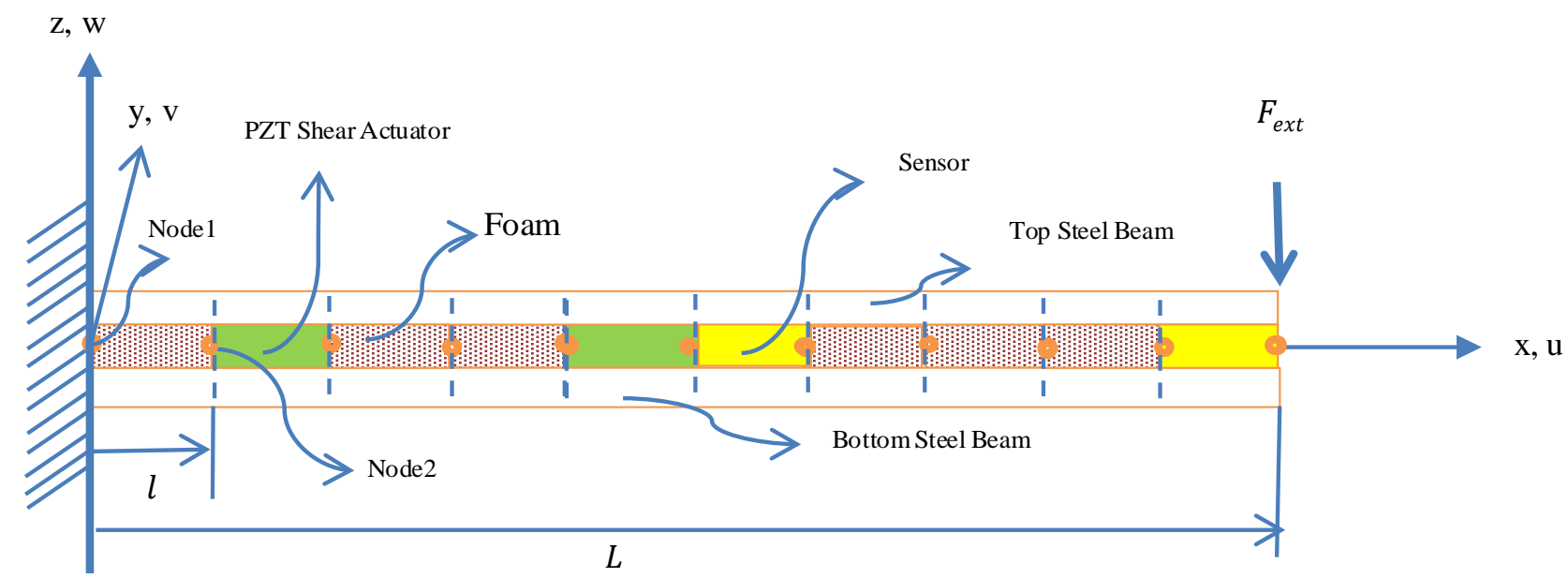

Figure 1. A three layered embedded beam (stacking sequence: steel/PZT or foam/steel) with MIMO.

For the parts having no PZT patch, the auxiliary space is being filled full with a material like obdurate foam. Again, there is a strong blend between foam and parent structure. Thus, embedded beam consists of slabs and a light weight core are effectively good in producing bending and shear action.

In analysis of embedded beam, the poling orientation of piezo patch in the axial direction. The displacement domain is based on first-order shear deformation theory (FSDT). The element is considered to have invariable elasticity modulus, moment of inertia, mass density, and length. The wiring capacitance is neglected between the sensor and signal conditioning device. The gain is assumed to be 100 for signal conditioning device.

Consider a beam having an element with two nodes. The longitudinal axis of the embedded beam element stays along $x$-axis and beam vibrates along $x-Z$ plane. The beam element has three degrees of freedom these are, axial displacement of the node $u$, transverse displacement of the node $w$, and bending rotation $\theta$. An auxiliary degree of freedom in the form of sensor voltage occurs. As sensor voltage is invariable through the element, the number of electrical degree of freedom is one. At each node, a bending moment and a transverse shear force act. The slope of the beam $\gamma(x)$ possesses two parts first one is the bending slope $\frac{\mathrm{d} w}{\mathrm{~d} x}$ and the second one is shear deformation angle $\phi(x)$.

\subsection{Equations of Motion}

The displacement of the beam is written as;

$$
u(x, z)=u_{0}(x)+z \theta(x, t) \text { and } w(x, z)=w_{0}(x)
$$

Strains are;

$$
\begin{aligned}
& \varepsilon_{x x}=\frac{\partial u_{0}}{\partial x}+z \frac{\partial \theta}{\partial x}, \varepsilon_{y y}=0, \varepsilon_{z z}=0 \\
& \gamma_{x y}=0, \gamma_{x y}=0, \gamma_{x z}=\frac{\partial u}{\partial x}+\frac{\partial w_{0}}{\partial x}=\theta+\frac{\partial w_{0}}{\partial x}
\end{aligned}
$$

The constitutive equations of the beam element are 


$$
\left[\begin{array}{c}
N_{x x} \\
M_{x x} \\
F_{x z}
\end{array}\right]=\left[\begin{array}{ccc}
A_{11} & B_{11} & 0 \\
B_{11} & B_{11} & 0 \\
0 & 0 & A_{55}
\end{array}\right]\left\{\begin{array}{c}
\frac{\partial u_{0}}{\partial x} \\
\frac{\partial \theta}{\partial x} \\
\theta+\frac{\partial w_{0}}{\partial x}
\end{array}\right\}-\left\{\begin{array}{l}
E_{11} \\
F_{11} \\
G_{55}
\end{array}\right\}
$$

where, $N_{x x}$ is in-plane force resultant in longitudinal direction, $M_{x x}$ is in-plane moment resultant in transverse direction and $F_{x z}$ is shear force resultant in transverse direction and they are given as

$$
N_{x x}=\int_{-\frac{h}{2}}^{\frac{h}{2}} b \sigma_{x x} \mathrm{~d} z, M_{x x}=\int_{-\frac{h}{2}}^{\frac{h}{2}} b \sigma_{x x} z \mathrm{~d} z, F_{x z}=\int_{-\frac{h}{2}}^{\frac{h}{2}} b \tau_{x z} \mathrm{~d} z
$$

Here, $b$ is beam width, $z$ is depth of material direction from beam reference plane, $h$ is the height of beam and piezoelectric patch. $A_{11}, B_{11}, D_{11}$ and $A_{55}$ are extensional, bending-extensional, bending and transverse shear stiffnesses and expounded by using lamination theory

$$
\begin{aligned}
& A_{11}=b \sum_{n=1}^{N}\left(\bar{Q}_{11}\right)_{n}\left(z_{n}-z_{n-1}\right), \\
& B_{11}=\frac{b}{2} \sum_{n=1}^{N}\left(\bar{Q}_{11}\right)_{n}\left(z_{n}^{2}-z_{n-1}^{2}\right), \\
& D_{11}=\frac{b}{3} \sum_{n=1}^{N}\left(\bar{Q}_{11}\right)_{n}\left(z_{n}^{3}-z_{n-1}^{3}\right), \\
& A_{55}=b \kappa \sum_{n=1}^{N}\left(\bar{Q}_{55}\right)_{n}\left(z_{n}-z_{n-1}\right)
\end{aligned}
$$

where, $z_{n}$ is the distance of $n^{\text {th }}$ lamina from longitudinal axis, $N$ is the total number of laminas, $\kappa$ is shear correction factor and $\bar{Q}_{11}, \bar{Q}_{55}$ are transformed reduced stiffnesses and given as

$$
\begin{gathered}
\bar{Q}_{11}=Q_{11} \cos ^{4} \psi+Q_{22} \sin ^{4} \psi+2\left(Q_{12}+2 Q_{66}\right) \sin ^{2} \psi \cos ^{2} \psi \\
\bar{Q}_{55}=G_{13} \cos ^{2} \psi+G_{23} \sin ^{2} \psi
\end{gathered}
$$

The angle $\psi$ is the angle between the fiber direction and $x$-axis of beam. Various material constants are obtained individually for steel, PZT and foam by relations listed in appendix. $E_{11}, F_{11}$ and $G_{55}$ are respectively actuator instigated piezoelectric axial force, bending moment owing to constrained actuator and shear force and given as

$$
\begin{gathered}
E_{11}=b \sum_{n=1}^{N_{\text {act }}}\left(\bar{Q}_{11}\right)_{n}^{a c t} V^{n}(x, t) d_{31}^{n}, \\
F_{11}=\frac{b}{2} \sum_{n=1}^{N_{\text {act }}}\left(\bar{Q}_{11}\right)_{n}^{a c t} V^{n}(x, t) d_{31}^{n}\left(z_{n+}^{a c t}-z_{n-}^{a c t}\right), \\
G_{55}=b \kappa \sum_{n=1}^{N_{a c t}}\left(\bar{Q}_{55}\right)_{n}^{a c t} V^{n}(x, t) d_{15}^{n},
\end{gathered}
$$

$E_{11}=F_{11}=0$, when $\mathrm{PZT}$ layer is oriented along longitudinal direction, 
$V^{n}(x, t)$ is applied voltage to $n^{\text {th }}$ actuator having thickness $\left(z_{n+}^{\text {act }}-z_{n-}^{\text {act }}\right)$ and $d_{31}^{n}, d_{15}^{n}$ are piezoelectric constants. $\left(\bar{Q}_{11}\right)_{n}^{a c t}$ and $\left(\bar{Q}_{55}\right)_{n}^{a c t}$ are the coefficients for actuator as evaluated using Equation (34), Equation (35), $N_{\text {act }}$ are the number of actuators.

Using Hamilton's principle (Dynamic version of principle of virtual work),

$$
\int_{0}^{T} \int_{0}^{l}(\delta U-\delta K+\delta W) \mathrm{d} x \mathrm{~d} t=0
$$

where $\delta U, \delta K$ and $\delta W$ correspond to virtual strain energy, virtual kinetic energy and virtual work done by external forces respectively and are given as

$$
\begin{gathered}
\delta U=N_{x x}\left(\frac{\partial \delta u}{\partial x}\right)+M_{x x}\left(\frac{\partial \delta \theta}{\partial x}\right)+F_{x x}\left(\theta+\frac{\partial \delta w}{\partial x}\right), \\
\delta K=\left(I_{1} \dot{u}+I_{2} \dot{\theta}\right) \delta \dot{u}+I_{1} \dot{w} \partial \dot{w}+\left(I_{2} \dot{u}+I_{3} \dot{\theta}\right) \delta \dot{\theta}, \\
\delta W=q_{0} \delta w
\end{gathered}
$$

where, $q_{0}$ is transverse load (equals to external force applied at the free end of beam).

$$
\begin{aligned}
& I_{i}(i=1,2,3) \text { are mass inertias of beam cross-section and are defined as } \\
& \qquad\left(I_{1}, I_{2}, I_{3}\right)=b \int_{-\frac{h}{2}}^{\frac{h}{2}} \rho\left(1, z, z^{2}\right) \mathrm{d} z
\end{aligned}
$$

Substituting the values of $\delta U, \delta K$ and $\delta W$ from Equations (40)-(42) in to Equation (39), we get equation of motion for general, unsymmetric piezoelectric laminated beam as per FSDT with shear deformation and rotary inertia as,

$$
\begin{gathered}
\frac{\partial}{\partial x}\left(A_{11} \frac{\partial u}{\partial x}+B_{11} \frac{\partial \theta}{\partial x}+E_{11}\right)=\frac{\partial}{\partial t}\left(I_{1} \dot{u}+I_{2} \dot{\theta}\right), \\
\frac{\partial}{\partial x}\left\{A_{55}\left(\theta+\frac{\partial w}{\partial x}\right)+G_{55}-P \frac{\partial w}{\partial x}\right\}=\frac{\partial}{\partial t}\left(I_{1} \dot{w}+q_{0}\right), \\
\frac{\partial}{\partial x}\left(B_{11} \frac{\partial u}{\partial x}+D_{11} \frac{\partial \theta}{\partial x}+F_{11}\right)-A_{55}\left(\theta+\frac{\partial w}{\partial x}\right)-G_{55}=\frac{\partial}{\partial t}\left(I_{2} \dot{u}+I_{3} \ddot{\theta}\right),
\end{gathered}
$$

For case of static loading with invariable beam properties. We have simplified form of equation of motion as

$$
\begin{gathered}
\frac{\partial}{\partial x}\left(A_{11} \frac{\partial u}{\partial x}+B_{11} \frac{\partial \theta}{\partial x}\right)=0 \\
\frac{\partial}{\partial x}\left\{A_{55}\left(\theta+\frac{\partial w}{\partial x}\right)\right\}=0 \\
\frac{\partial}{\partial x}\left(B_{11} \frac{\partial u}{\partial x}+D_{11} \frac{\partial \theta}{\partial x}+F_{11}\right)-A_{55}\left(\theta+\frac{\partial w}{\partial x}\right)-G_{55}=0
\end{gathered}
$$

For the solution of unknowns, the degree of polynomial used for axial displacement, $u$ and bending rotation, $\theta$ must be one order lower than that used for transverse displacement, $w$ to satisfy the compatibility. Here we used 
quadratic function for $u, \theta$ and cubic function for $w$. Let the solution be assumed as

$$
\begin{gathered}
w=p_{1}+p_{2} x+p_{3} x^{2}+p_{4} x^{3} ; \\
\theta=q_{1}+q_{2} x+q_{3} x^{2}, \\
u=r_{1}+r_{2} x+r_{3} x^{2},
\end{gathered}
$$

The boundary conditions are

$$
\begin{aligned}
& \text { at } x=0: w=w_{1}, \theta=\theta_{1}, u=u_{1} \\
& \text { at } x=l: w=w_{2}, \theta=\theta_{2}, u=u_{2}
\end{aligned}
$$

Using the boundary conditions in Equations (50)-(52), the unknown coefficients $p_{i}, q_{j}$ and $r_{j}$ are determined. Substituting the evaluated unknowns into Equations (50)-(52) and arranging them into matrix form, we obtain expressions for $w, u$ and $\theta$ in terms of shape functions and nodal displacements.

$$
[w]=\left[N_{w}\right]\left[\begin{array}{c}
u_{1} \\
w_{1} \\
\theta_{1} \\
u_{2} \\
w_{2} \\
\theta_{2}
\end{array}\right],[u]=\left[N_{u}\right]\left[\begin{array}{c}
u_{1} \\
w_{1} \\
\theta_{1} \\
u_{2} \\
w_{2} \\
\theta_{2}
\end{array}\right],[\theta]=\left[N_{\theta}\right]\left[\begin{array}{c}
u_{1} \\
w_{1} \\
\theta_{1} \\
u_{2} \\
w_{2} \\
\theta_{2}
\end{array}\right]
$$

where, $\left[N_{w}\right],\left[N_{u}\right]$ and $\left[N_{\theta}\right]$ are modal shape functions due to $w, u$ and $\theta$ which are given as

$$
\begin{aligned}
& {\left[N_{w}\right]=\left[\begin{array}{llllll}
N_{1} & N_{2} & N_{3} & N_{4} & N_{5} & N_{6}
\end{array}\right]} \\
& {\left[N_{u}\right]=\left[\begin{array}{llll}
N_{7} & N_{8} & N_{9} & N_{10}
\end{array}\right]} \\
& {\left[N_{\theta}\right]=\left[\begin{array}{llll}
N_{11} & N_{12} & N_{13} & N_{14}
\end{array}\right]}
\end{aligned}
$$

Writing these three shape functions in matrix form, the relations between inertial forces vector $\mathbb{N}$ and nodal displacement vector $\boldsymbol{d}$ as

$$
[\mathbb{N}]=\left[\begin{array}{cccccc}
N_{1} & N_{2} & N_{3} & N_{4} & N_{5} & N_{6} \\
0 & N_{7} & N_{8} & 0 & N_{9} & N_{10} \\
0 & N_{11} & N_{12} & 0 & N_{13} & N_{14}
\end{array}\right]\left\{\begin{array}{c}
u_{1} \\
w_{1} \\
\theta_{1} \\
u_{2} \\
w_{2} \\
\theta_{2}
\end{array}\right\}
$$

The shape function elements in Equations (55)-(57) are presented in appendix.

The mass matrix of beam element is given as

$$
\left[M^{\text {beam }}\right]=\int_{0}^{l}[\mathbb{N}]^{\mathrm{T}}[I][\mathbb{N}] \mathrm{d} x
$$

where, $[I]$ is the inertia vector and given as

$$
[I]=\left[\begin{array}{ccc}
I_{1} & 0 & I_{2} \\
0 & I_{1} & 0 \\
I_{2} & 0 & I_{3}
\end{array}\right]
$$


The mass matrix for beam element is finally given as

$$
\left[M^{\text {beam }}\right]=\left[\begin{array}{llllll}
m_{11} & m_{12} & m_{13} & m_{14} & m_{15} & m_{16} \\
m_{21} & m_{22} & m_{23} & m_{24} & m_{25} & m_{26} \\
m_{31} & m_{32} & m_{33} & m_{34} & m_{35} & m_{36} \\
m_{41} & m_{42} & m_{43} & m_{44} & m_{45} & m_{46} \\
m_{51} & m_{52} & m_{53} & m_{54} & m_{55} & m_{56} \\
m_{61} & m_{62} & m_{63} & m_{64} & m_{65} & m_{66}
\end{array}\right]
$$

$\left[M^{\text {beam }}\right]$ is a symmetric local mass matrix of size $6 \times 6$ for a beam element, its coefficients are given in the appendix.

The stiffness matrix of beam element is given as

$$
\left[K^{\text {beam }}\right]=\int_{0}^{l}[\mathbb{R}]^{T}[D][\mathbb{R}] A \mathrm{~d} x
$$

where $A$ is the area of beam cross-section and

$$
[\mathbb{R}]=\frac{\mathrm{d}[\mathbb{N}]}{\mathrm{d} x} \text { and }[D]=\left[\begin{array}{ccc}
A_{11} & B_{11} & 0 \\
B_{11} & B_{11} & 0 \\
0 & 0 & A_{55}
\end{array}\right]
$$

Finally, the stiffness matrix of the beam element is given as

$$
\left[K^{\text {beam }}\right]=\left[\begin{array}{llllll}
k_{11} & k_{12} & k_{13} & k_{14} & k_{15} & k_{16} \\
k_{21} & k_{22} & k_{23} & k_{24} & k_{25} & k_{26} \\
k_{31} & k_{32} & k_{33} & k_{34} & k_{35} & k_{36} \\
k_{41} & k_{42} & k_{43} & k_{44} & k_{45} & k_{46} \\
k_{51} & k_{52} & k_{53} & k_{54} & k_{55} & k_{56} \\
k_{61} & k_{62} & k_{63} & k_{64} & k_{65} & k_{66}
\end{array}\right]
$$

$\left[K^{\text {beam }}\right]$ is a symmetric local stiffness matrix of size $6 \times 6$ for a beam element, its coefficients are given in appendix.

The mass matrix and stiffness matrix of the regular beam are obtained by placing foam core in between two laminas of steel. Similarly, a piezoelectric patch is used in place of foam between two laminas to obtain piezoelectric beam element.

\subsection{Equation of Sensing Component}

Sensor works on direct piezoelectric effect, which is used to evaluate the output charge developed due to straining of the structure. The electric displacement produced by the sensor is directly proportional to strain rate. The charge $q(t)$ appeared on piezoelectric sensor surface is given by Gauss law as

$$
q(t)=\int_{A_{P Z}} D_{Z} \mathrm{~d} A_{P Z}
$$

where, $D_{z}$ is electric displacement in thickness direction and $A_{P Z}$ is the area of shear PZT patch. If poling is done along the thickness direction having electrodes on top and bottom surfaces, the electric displacement is given as

$$
D_{z}=\bar{Q}_{55} d_{15} \gamma_{x z}=e_{15} \gamma_{x z}
$$


Using Equation (66) into Equation (65), we get

$$
q(t)=\int_{A_{P Z}} e_{15}\left(\theta+\frac{\partial w_{0}}{\partial x}\right) \mathrm{d} A_{P Z}
$$

Solving Equation (67), we get

$$
q(t)=e_{15} b_{b} \frac{6 \lambda}{-12 \lambda+l_{P Z}^{2}}\left[\begin{array}{llllll}
0 & 2 & -l_{P Z} & 0 & -2 & -l_{P Z}
\end{array}\right]\left\{\begin{array}{l}
u_{1} \\
w_{1} \\
\theta_{1} \\
u_{2} \\
w_{2} \\
\theta_{2}
\end{array}\right\}
$$

Here, $\left\{\begin{array}{l}u_{1} \\ w_{1} \\ \theta_{1} \\ u_{2} \\ w_{2} \\ \theta_{2}\end{array}\right\}=\boldsymbol{d}$ is the nodal displacement vector, $b_{b}$ is the width of the beam, $\lambda=\frac{D_{11}}{A_{55}}\left(\mu \frac{B_{11}}{D_{11}}-1\right)$ and $\mu=\frac{B_{11}}{A_{11}}$.

The current induced by the sensor is

$$
\begin{gathered}
i(t)=\frac{\mathrm{d} q(t)}{\mathrm{d} t} \\
i(t)=e_{15} b_{b} \frac{6 \lambda}{-12 \lambda+l_{P Z}^{2}}\left[\begin{array}{llllll}
0 & 2 & -l_{P Z} & 0 & -2 & -l_{P Z}
\end{array}\right] \dot{\boldsymbol{d}}
\end{gathered}
$$

With the use of signal conditioning device this current is converted into open circuit sensor voltage $V^{\text {sen }}(t)$ and applied to actuator with controller gain

$$
\begin{aligned}
& V^{s e n}(t)=G_{s c} i(t) \\
& V^{s e n}(t)=G_{s c} e_{15} b_{b} \frac{6 \lambda}{-12 \lambda+l_{P Z}^{2}}\left[\begin{array}{llllll}
0 & 2 & -l_{P Z} & 0 & -2 & -l_{P Z}
\end{array}\right] \dot{\boldsymbol{d}} \\
& V^{\text {sen }}(t)=\boldsymbol{b}^{\mathrm{T}} \dot{\boldsymbol{d}}
\end{aligned}
$$

where, $\dot{\boldsymbol{d}}$ is the strain rate and $\boldsymbol{b}^{\mathrm{T}}$ is a constant vector of $(1 \times 6)$ size for a double node beam element which depends on sensor type, its properties and its location in embedded structure and described as

$$
\boldsymbol{b}^{\mathrm{T}}=e_{15} b_{b} G_{s c} \frac{6 \lambda}{-12 \lambda+l_{P Z}^{2}}\left[\begin{array}{llllll}
0 & 2 & -l_{P Z} & 0 & -2 & -l_{P Z}
\end{array}\right]
$$

The actuator input voltage is

$$
\begin{gathered}
V^{a c t}(t)=K \times V^{s e n}(t) \\
V^{a c t}(t)=K \times G_{s c} e_{15} b_{b} \frac{6 \lambda}{-12 \lambda+l_{P Z}^{2}}\left[\begin{array}{llllll}
0 & 2 & -l_{P Z} & 0 & -2 & -l_{P Z}
\end{array}\right] \dot{\boldsymbol{d}}
\end{gathered}
$$




\subsection{Equation of Actuating Component}

Actuator works on converse piezoelectric effect which is used to evaluate the straining effect caused due to actuator. The strain produced in PZT patch is directly proportional to electric potential applied to lamina and is given as

$$
\gamma_{x Z}^{P Z} \propto E^{a c t}
$$

where, $\gamma_{x z}^{P Z}$ is shear strain in in PZT lamina and $E^{a c t}$ is the electric potential applied to actuator, which is,

$$
E^{a c t}=\frac{V^{a c t}}{t_{P Z}}
$$

where, $t_{P Z}$ is the thickness of PZT lamina. From Equation (77)

$$
\gamma_{x Z}^{P Z}=d_{15} E^{a c t}
$$

Shear stress in PZT lamina is given as

$$
\tau_{x z}^{P Z}=G \gamma_{x z}^{P Z}
$$

where, $G$ is the modulus of rigidity. Substituting the values of $\gamma_{x z}^{P Z}$ into Equation (79), we get

$$
\tau_{x z}^{P Z}=G d_{15} E^{a c t}
$$

Using Equation (78) in Equation (81), we obtain

$$
\tau_{x Z}^{P Z}=G d_{15} \frac{V^{a c t}}{t_{P Z}}
$$

Due to this stress, bending moments are incorporated into the beam at the nodes. The moment $M^{\text {act }}$ acting on beam element is obtained by integration of shear stress through structural thickness in Equation (82). Finally

$$
M^{a c t}=G d_{15} V^{a c t} \tilde{h}
$$

Or we may write

$$
M^{a c t}=G d_{15} K \boldsymbol{b}^{\mathrm{T}} \dot{\boldsymbol{d}} \tilde{h}
$$

Here, $\tilde{h}=\frac{t_{\text {act }}+t_{\text {beam }}}{2}$ is the distance between neutral axis of beam and neutral axis of PZT patch.

The control force $f^{\text {ctrl }}$ produced by actuator and applied to beam is obtained from Equation (84) as

$$
f^{c t r l}=G d_{15} \tilde{h} \int_{0}^{l_{P Z}} N_{\theta} \mathrm{d} x V^{a c t}(t)
$$

Or simplified as

$$
f^{c t r l}=\boldsymbol{g} V^{a c t}(t)
$$

where, $\boldsymbol{g}$ is a constant vector of size $(6 \times 1)$ for a double node beam element and depends on actuator location and type. The total force vector in existence of any external force vector is 


$$
f^{\text {total }}=f^{e x t}+f^{c t r l}
$$

where, $f^{\text {ext }}$ representing the external disturbance vector.

\subsection{Dynamic Equation of Smart Structure for A MIMO Model}

The dynamic equation for the smart structure is obtained by using both the regular beam element and the piezoelectric beam element. The mass and stiffness matrices of the regular beam and piezoelectric beam element are known as local mass and stiffness matrices and give only the mass and stiffness matrices of only one finite element. The mass and stiffness matrices of entire beam i.e. divided into 10 finite elements are obtained by assembling the local matrices by applying finite element technique and resulting matrices are called global mass matrix $\left[M^{G}\right]$ and global stiffness matrix $\left[K^{G}\right]$. The mass and stiffness matrices $\left[M^{G}\right](20 \times 20)$ and $\left[K^{G}\right](20 \times 20)$ of dynamic equation of smart structure have both sensor and actuator masses and stiffnesses.

The equation of motion of the smart structure and sensor output is

$$
\begin{gathered}
M^{G} \ddot{\boldsymbol{d}}+K^{G} \boldsymbol{d}=f^{e x t}+f^{c t r l 1}+f^{c t r l 2}=f^{e x t}+f^{c t r l i}=f^{\text {total }} \\
\& y_{i}(t)=V^{\operatorname{sen} i}(t)=\boldsymbol{b}_{i}^{\mathrm{T}} \dot{\boldsymbol{d}}, \quad(i=1,2)
\end{gathered}
$$

The mass and stiffness matrices of the beam in the equation of system (64) can be changed by varying the location of PZT patch on beam and by varying the number of regular and piezoelectric beam elements. The generalized coordinates are introduced into Equation (64) with a transformation $\boldsymbol{d}=\boldsymbol{T a}$, in order to reduce it further so the resulting equation showing the dynamics of desired vibratory modes. Here $\boldsymbol{T}$ is a modal matrix containing the eigenvectors showing the desired number of modes of vibration. This procedure is applied to derive the uncoupled equations governing forced vibration in terms of principal coordinates by inducing linear transformation between generalized coordinates $\boldsymbol{d}$ and principal coordinates $\boldsymbol{a}$ and hence decoupling into equations related to each mode. Using the transformation $\boldsymbol{d}=\boldsymbol{T} \boldsymbol{a}$. Equation (88), Equation (89) become

$$
\begin{aligned}
& M^{G} \boldsymbol{T} \ddot{\boldsymbol{a}}+K^{G} \boldsymbol{T a}=f^{e x t}+f^{c t r l i}=f^{\text {total }} \\
& \& \quad y_{i}(t)=V^{\text {sen } i}(t)=\boldsymbol{b}_{i}^{\mathrm{T}} \dot{\boldsymbol{d}}=\boldsymbol{b}_{i}^{\mathrm{T}} \boldsymbol{T} \dot{\boldsymbol{a}}
\end{aligned}
$$

Premultiplying Equation (90) by $\boldsymbol{T}^{\mathrm{T}}$, we get

$$
\boldsymbol{T}^{\mathrm{T}} \boldsymbol{M}^{G} \boldsymbol{T} \ddot{\boldsymbol{a}}+\boldsymbol{T}^{\mathrm{T}} K^{G} \boldsymbol{T a}=\boldsymbol{T}^{\mathrm{T}} f^{e x t}+\boldsymbol{T}^{\mathrm{T}} f^{\text {ctrl } i}=\boldsymbol{T}^{\mathrm{T}} f^{\text {total }}
$$

Which may be written as

$$
M \ddot{a}+K \boldsymbol{a}=F^{e x t}+F^{c t r l i}
$$

where, $M=\boldsymbol{T}^{\mathrm{T}} M^{G} \boldsymbol{T}$ and $K=\boldsymbol{T}^{\mathrm{T}} K^{G} \boldsymbol{T}$ are called the generalized mass and stiffness matrices.

The generalized external force vector

$$
F^{e x t}=\boldsymbol{T}^{\mathrm{T}} f^{e x t}=\boldsymbol{T}^{\mathrm{T}} \boldsymbol{f r}(t)
$$

where, $r(t)$ is external force input to beam. 
The generalized control force vector

$$
F^{c t r l i}=\boldsymbol{T}^{\mathrm{T}} f^{c t r l i}=\boldsymbol{T}^{\mathrm{T}} \boldsymbol{g}_{i} V^{a c t}(t)=\boldsymbol{T}^{\mathrm{T}} \boldsymbol{g}_{i} u_{i}(t)
$$

where, $r(t)$ is control force input to beam.

The structural modal damping matrix $C$ by using Rayleigh proportional damping as

$$
C=\alpha M+\beta K
$$

where, $\alpha, \beta$ are frictional damping constants and structural damping constant in $C$.

The dynamic equation and sensor output of smart structure is finally

$$
\begin{aligned}
M \ddot{\boldsymbol{a}}+C \dot{\boldsymbol{a}}+K \boldsymbol{a} & =F^{e x t}+F^{c t r l i} \\
\& & y_{i}(t)=V^{\text {sen i }}(t)=\boldsymbol{b}_{i}^{\mathrm{T}} \dot{\boldsymbol{d}}=\boldsymbol{b}_{i}^{\mathrm{T}} \boldsymbol{T} \dot{\boldsymbol{a}}
\end{aligned}
$$

\subsection{State Space Formulation for A MIMO Model}

Here in the present case of actively controlled cantilever beam only first three vibratory modes are controlled since more energy is stored in lower order modes as similar to lower order Fourier components are larger in magnitude and the higher frequency components are smaller as the harmonics increase in number [38]. The state space model for first three vibratory modes can be obtained as, let $\boldsymbol{a}=\boldsymbol{x}$ where,

$$
\boldsymbol{a}=\left\{\begin{array}{l}
a_{1} \\
a_{2} \\
a_{3}
\end{array}\right\}=\left\{\begin{array}{l}
x_{1} \\
x_{2} \\
x_{3}
\end{array}\right\}=\boldsymbol{x}
$$

Now

$$
\dot{\boldsymbol{a}}=\dot{\boldsymbol{x}}=\left\{\begin{array}{l}
\dot{a}_{1} \\
\dot{a}_{2} \\
\dot{a}_{3}
\end{array}\right\}=\left\{\begin{array}{l}
\dot{x}_{1} \\
\dot{x}_{2} \\
\dot{x}_{3}
\end{array}\right\}=\left\{\begin{array}{c}
x_{4} \\
x_{5} \\
x_{6}
\end{array}\right\} \text { and } \ddot{\boldsymbol{a}}=\ddot{\boldsymbol{x}}=\left\{\begin{array}{c}
\dot{x}_{4} \\
\dot{x}_{5} \\
\dot{x}_{6}
\end{array}\right\}
$$

Using Equation (94), Equation (95), Equation (99) and Equation (100) in to Equation (97), the dynamic equation of the smart structure with 3 vibratory modes

$$
M\left\{\begin{array}{l}
\dot{x}_{4} \\
\dot{x}_{5} \\
\dot{x}_{6}
\end{array}\right\}+C\left\{\begin{array}{c}
x_{4} \\
x_{5} \\
x_{6}
\end{array}\right\}+K\left\{\begin{array}{c}
x_{1} \\
x_{2} \\
x_{3}
\end{array}\right\}=F^{e x t}+F^{c t r l ~ i}
$$

Which can be further simplified as

$$
\left\{\begin{array}{l}
\dot{x}_{4} \\
\dot{x}_{5} \\
\dot{x}_{6}
\end{array}\right\}=-M^{-1} K\left\{\begin{array}{l}
x_{4} \\
x_{5} \\
x_{6}
\end{array}\right\}-M^{-1} C\left\{\begin{array}{c}
x_{1} \\
x_{2} \\
x_{3}
\end{array}\right\}+M^{-1} F^{e x t}+M^{-1} F^{c t r l ~ i}
$$

In state space form

$$
\begin{gathered}
\dot{\boldsymbol{x}}=\boldsymbol{A} x(t)+\boldsymbol{B} u(t)+\boldsymbol{E} r(t) \\
\boldsymbol{y}(t)=\boldsymbol{C}^{\mathrm{T}} x(t)+\boldsymbol{D} u(t)
\end{gathered}
$$


As,

$$
\begin{aligned}
\left\{\begin{array}{l}
\dot{x}_{1} \\
\dot{x}_{2} \\
\dot{x}_{3} \\
\dot{x}_{4} \\
\dot{x}_{5} \\
\dot{x}_{6}
\end{array}\right\}= & {\left[\begin{array}{cc}
0 & I \\
-M^{-1} K & -M^{-1} C
\end{array}\right]\left\{\begin{array}{l}
x_{1} \\
x_{2} \\
x_{3} \\
x_{4} \\
x_{5} \\
x_{6}
\end{array}\right\}+\left[\begin{array}{cc}
0 & I \\
M^{-1} \boldsymbol{T}^{\mathrm{T}} \boldsymbol{g}_{1} & M^{-1} \boldsymbol{T}^{\mathrm{T}} \boldsymbol{g}_{2}
\end{array}\right]\left[\begin{array}{l}
u_{1}(t) \\
u_{2}(t)
\end{array}\right] } \\
& +\left[\begin{array}{cc}
0 \\
M^{-1} \boldsymbol{T}^{\mathrm{T}} \boldsymbol{f}
\end{array}\right] r(t)
\end{aligned}
$$

$$
\left[\begin{array}{l}
y_{1} \\
y_{2}
\end{array}\right]=\left[\begin{array}{ll}
0 & \boldsymbol{b}_{1}^{\mathrm{T}} \boldsymbol{T} \\
0 & \boldsymbol{b}_{2}^{\mathrm{T}} \boldsymbol{T}
\end{array}\right]\left\{\begin{array}{l}
x_{1} \\
x_{2} \\
x_{3} \\
x_{4} \\
x_{5} \\
x_{6}
\end{array}\right\}
$$

where, $\boldsymbol{A}$ is state matrix, $\boldsymbol{B}$ is input matrix, $\boldsymbol{C}$ is output matrix, $\boldsymbol{D}$ is transmission matrix, $\boldsymbol{E}$ is external load matrix coupling the disturbance to the system and all are in continuous time model of LTI system. The size of matrices $\boldsymbol{A}, \boldsymbol{B}, \boldsymbol{C}, \boldsymbol{E}$ and $\boldsymbol{T}$ are $(6 \times 6),(6 \times 2),(2 \times 6),(6 \times 1)$ and $(20 \times 3)$ with $\boldsymbol{D}$ being a null matrix.

\section{Simulation for Controllers for Smart Beams with MIMO Using Embedded Piezo}

A cantilever beam of proposed parameters as given in Table 1, the piezo material properties in Table 2 and material constants in Table 3. The beam is divided into 10 finite elements and shear piezo are embedded into parent structure as sensors and actuators as presented in Figure 1. The actuators are placed in between two thick steel beams at FE position 2 and 5, while the sensors are placed at FE location 6 and 10, hence developing a single MIMO system with 2 inputs and 2 outputs.

Table 1. Properties of the steel cantilever Timoshenko beam.

\begin{tabular}{ccc}
\hline Parameter & Symbol & Numerical Value \\
\hline Length $(\mathrm{m})$ & $L$ & 0.4 \\
Width $(\mathrm{m})$ & $b_{b}$ & 0.02 \\
$\begin{array}{c}\text { Thickness of the top layer and bottom } \\
\text { steel beam layers }(\mathrm{m})\end{array}$ & $t_{\text {beam }}$ & 0.01 \\
Young's Modulus $(\mathrm{GPa})$ & $E_{s}$ & 210 \\
Density $\left(\mathrm{kg} / \mathrm{m}^{3}\right)$ & $\rho_{b}$ & 8030 \\
Damping Constants & $\alpha, \beta$ & $0.001,0.0001$
\end{tabular}


Table 2. Properties of the piezoelectric shear sensor and actuator when the beam is divided into 10 finite elements.

\begin{tabular}{ccc}
\hline Parameter & Symbol & Numerical Value \\
\hline Length $(\mathrm{m})$ & $l_{P Z}$ & 0.04 \\
Width $(\mathrm{m})$ & $b_{P Z}$ & 0.02 \\
Thickness $(\mathrm{m})$ & $t_{a}, t_{s}$ & 0.001 \\
Young's Modulus $(\mathrm{GPa})$ & $E_{P Z}$ & 84.1 \\
Density $\left(\mathrm{kg} / \mathrm{m}^{3}\right)$ & $\rho_{P Z}$ & 7900 \\
Piezoelectric strain constant & $d_{31}$ & $-274.8 \times 10^{-12}$ \\
\hline
\end{tabular}

Table 3. Material properties and constants.

\begin{tabular}{cccc}
\hline Material Constants & PZT & Steel & Foam \\
\hline$G_{12}\left(\mathrm{~N} / \mathrm{m}^{2}\right)$ & $24.8 \times 10^{9}$ & $78.7 \times 10^{9}$ & $9.99 \times 10^{6}$ \\
$G_{13}\left(\mathrm{~N} / \mathrm{m}^{2}\right)$ & $24.8 \times 10^{9}$ & $78.7 \times 10^{9}$ & $9.99 \times 10^{6}$ \\
$G_{23}\left(\mathrm{~N} / \mathrm{m}^{2}\right)$ & $24.8 \times 10^{9}$ & $78.7 \times 10^{9}$ & $9.99 \times 10^{6}$ \\
$d_{31}(\mathrm{~m} / \mathrm{V})$ & $-0.166 \times 10^{-9}$ & - & - \\
$d_{15}(\mathrm{~m} / \mathrm{V})$ & $1.34 \times 10^{-9}$ & - & - \\
$Q_{11}\left(\mathrm{~N} / \mathrm{m}^{2}\right)$ & $68.4 \times 10^{9}$ & $211 \times 10^{9}$ & $85.5 \times 10^{6}$ \\
$Q_{22}\left(\mathrm{~N} / \mathrm{m}^{2}\right)$ & $68.4 \times 10^{9}$ & $211 \times 10^{9}$ & $85.5 \times 10^{6}$ \\
$Q_{12}\left(\mathrm{~N} / \mathrm{m}^{2}\right)$ & $12.6 \times 10^{9}$ & $2.88 \times 10^{9}$ & $75.6 \times 10^{6}$ \\
$Q_{66}\left(\mathrm{~N} / \mathrm{m}^{2}\right)$ & $12.6 \times 10^{9}$ & $78.7 \times 10^{9}$ & $9.99 \times 10^{6}$ \\
\hline
\end{tabular}

The MIMO model achieved by using the TBT, piezoelectric coupling, FE modeling and state space approach by taking first three vibratory modes into consideration. An external impulsive force $f^{\text {ext }}$ of $10 \mathrm{~N}$ is employed for $60 \mathrm{~ms}$ at the free end of the cantilever beam. There are three inputs to the system, the first one is the external force $f^{\text {ext }}$ responsible for the disturbance. Other inputs are the control inputs $u_{i}(i=1,2)$ to actuators by the controller.

The control strategy presented in this monograph is implemented to design a multi-rate output feedback based discrete sliding mode controller to attenuate the first three modes of vibration of a cantilever beam by using smart structure approach of smart embedded beam with MIMO.

The performance of the model with multiple inputs and multiple outputs for active vibration attenuation by performing simulations in MATLAB $^{\circledR}$ and analyzing different responses. The discrete sliding mode controller is designed and implemented to MIMO system. The responses viz. control inputs, sensor outputs, and switching are demonstrated in included Figure (2a), Figure (2b), Figure (3a), Figure ( $3 b$ ) and Figure (4a), Figure (4b) respectively. The values are obtained to be $\delta_{\zeta}=1 \times 10^{-10}\left[\begin{array}{ll}37.56 & 44.93\end{array}\right]^{\mathrm{T}}$ and $\delta_{o}=1 \times 10^{-10}\left[\begin{array}{ll}566.85 & 52.29\end{array}\right]^{\mathrm{T}}$ 


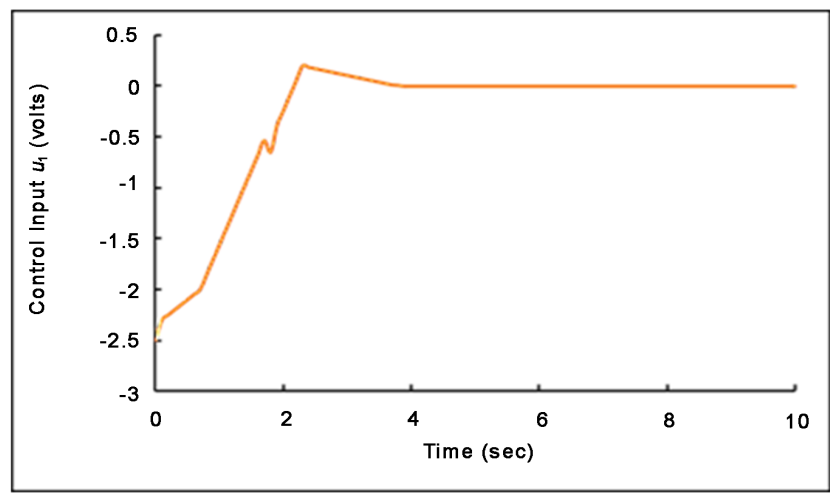

(a)

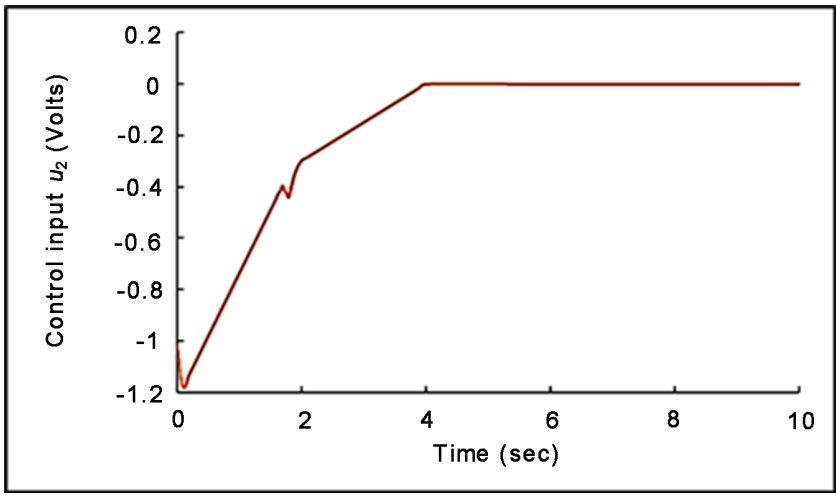

(b)

Figure 2. Plot for control input for embedded smart cantilever beam.

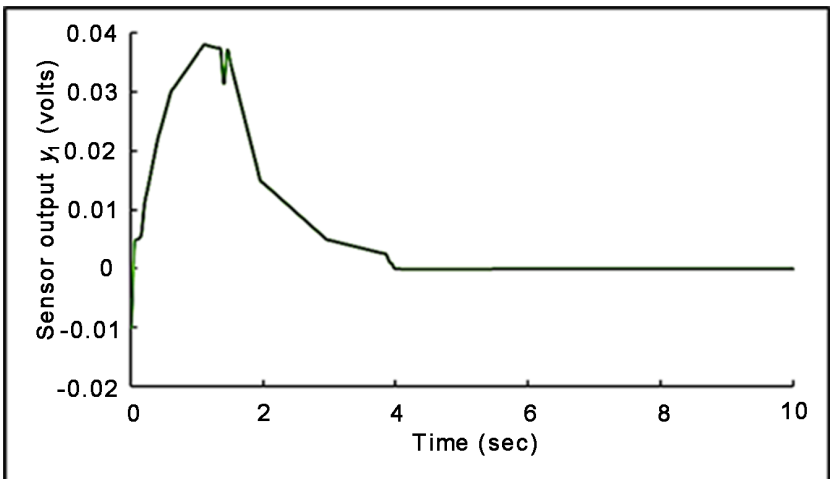

(a)

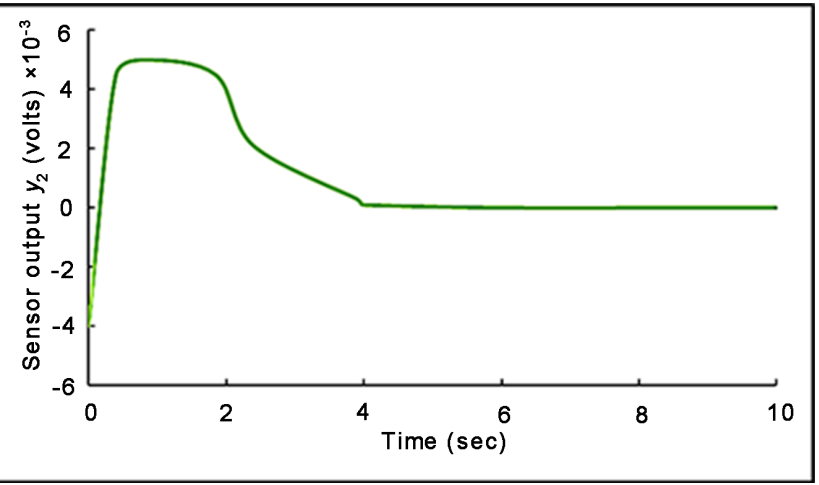

(b)

Figure 3. Plot for sensor output for embedded smart cantilever beam.

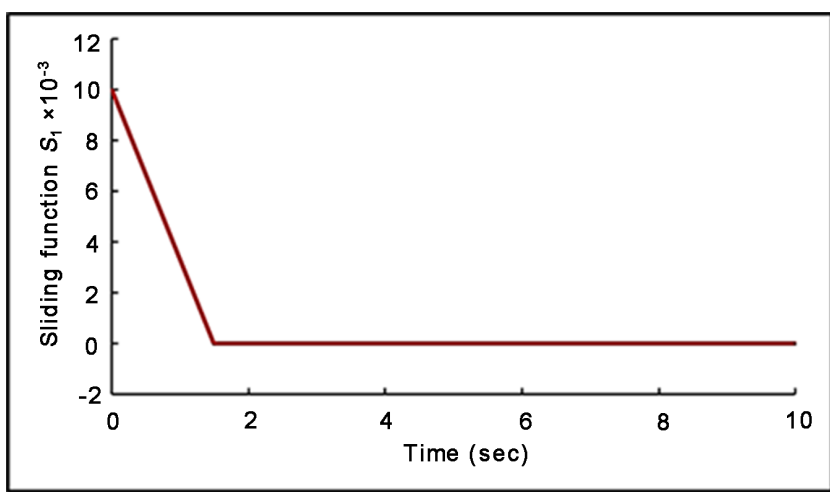

(a)

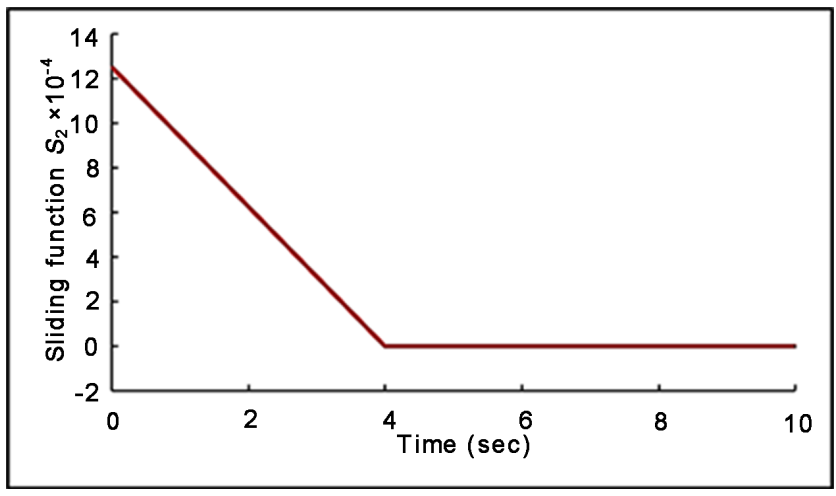

(b)

Figure 4. Plot for Sliding functions for embedded smart cantilever beam.

\section{Elucidation of Results}

DSM controllers have designed to control first three modes of vibration of a flexible cantilever beam modelled based on Timoshenko beam theory. New active vibration control scheme to suppress the vibrations of MIMO model has developed. The actuators are located at $2^{\text {nd }}$ and $5^{\text {th }} \mathrm{FE}$ positions while sensors are set at $6^{\text {th }}$ and $10^{\text {th }} \mathrm{FE}$ position to form the embedded smart cantilever beam with 
10 finite elements. The piezo crystals are located in the central core at prescribed locations, rest core filled with rigid foam, and this central core is sandwiched between two regular steel beams as shown in Figure 1.

It is obvious from previous works that the control is more persuasive at the root with the sensor output voltage is significantly more due to the substantial dispensation of the bending moment near the firm end for the rudimentary mode, thus provoking a stupendous strain rate and the susceptibility of the sensor/actuator duo rely on its placement in the beam and the vibration attributes of the system precarious on collocation of the piezo pair and also on some other numerous facet viz. the gain of amplifier employed, the mode number and the placement of piezo patches at the nodal points from fixed end [28]. Modelling a smart structure inclusive of sensor/actuator mass and stiffness and by altering its orientation in the beam from the free end to the fixed end acquaint an ample modification in the system's structural response attributes. Sensor voltage is lower when the piezo patch is imposed at the free end due to the exiguous strain rate and hence demand more control endeavor. MIMO control is superior over SISO control due to its multifarious interactions of input and output and all-inclusive control endeavor needed by MIMO controller is less than SISO controller and also placing the piezo at two distinct FE locations on the beam establishing the significant modification in the system structural traits than placing it lonely at a location. [39].

The multirate output feedback dependent DSMC strategy are more harmonious as compared to the other control approaches viz. periodic output feedback (POF) and fast output sampling (FOS) controllers [40]. The multirate output feedback based DSMC policy are more episodic as compared to the other control techniques. In discrete quasi sliding mode control (DQSMC) with output samples, there is a necessity of switching function for control and hence engendering some chattering phenomenon [41], while control strategy presented in the present article is the MROF based DSMC technique obtained from Bartoszewicz's law does not demand any use of switching function and provides control input directly in form of past control data and past samples. The system responds well in closed loop and does not manifest inexpedient chattering phenomenon. MROF based DSMC employ the signum function in the control input and the control is computed from the immediate past control value and the past control output samples. The fractious system takes an extended time to damp out the oscillations in contrast to the system with the designed sliding mode control input means without control the transient response was preeminent and with control, the vibrations are quashed.

From simulation results, it can be inferred that sensor output at FE 6 is more than sensor output at FE 10 by approximately 10 times due to its high strain rate at FE 6 as compared at FE 10 and also the control input are approximately 10 times smaller in case of MROF as compared to SISO case [41]. In case of MROF technique, the states of the system are needed neither for switching function assessment nor for the feedback denotation. DSMC algorithm are computationally 
unpretentious, ensures better enduringness, brisk convergence and exalted steady state authenticity of the system. The technique used is more feasible as the output being used rather than states.

Hence, it can be concluded that the multivariable control is best among all the models due to its multilevel interactions on both input and output. A MIMO model furnishes excellent energy distribution and even good administration of actuation forces and minimal requirement of control forces as compared to SISO model for the case of smart cantilever beam with embedded sensors and actuators.

\section{References}

[1] Culshaw, B. (1996) Smart Structures and Materials. Artech House, Norwood, Boston.

[2] Tani, J., Takaga, T. and Qiu, J. (1998) Intelligent Material System: Application of Functional Materials. Applied Mechanics Reviews, 51, 505-521. https://doi.org/10.1115/1.3099019

[3] Bailey, T. and Hubbard, J. (1985) Distributed Piezoelectric Polymers Active Vibration Control of a Cantilever Beam. Journal of Guidance, Control and Dynamics, 8 , 605-611. https://doi.org/10.2514/3.20029

[4] Crawley, E.F. and Luis, J.D. (1987) Use of Piezoelectric Actuators as Elements of Intelligent Structures. AIAA Journal, 25, 1373-1385. https://doi.org/10.2514/3.9792

[5] Tzou, H.S. and Tseng, C.I. (1990) Distributed Piezoelectric Sensor/Actuator Design for Dynamic Measurement/Control of Distributed Parameter Systems. Journal of Sound and Vibration, 138, 17-34. https://doi.org/10.1016/0022-460X(90)90701-Z

[6] Baz, A. and Poh, S. (1988) Performance of an Active Control System with Piezoelectric Actuators. Journal of Sound and Vibration, 126, 327-343. https://doi.org/10.1016/0022-460X(88)90245-3

[7] Ha, S.K., Keilers, C. and Chang, F.K. (1992) Finite Element Analysis of Composite Structures Containing Distributed Piezoceramic Sensors and Actuators. AIAA Journal, 30, 772-780. https://doi.org/10.2514/3.10984

[8] Hanagud, S., Obal, M.W. and Carlise, A.J. (1992) Optimal Vibration Control by the Use of Piezoceramic Sensors and Actuators. Journal of Guidance, Control and Dynamics, 15, 1199-1206. https://doi.org/10.2514/3.20969

[9] Rao, S.S. and Sunar, M. (1993) Analysis of Distributed Thermopiezoelastic Sensors and Actuators in Advanced Intelligent Structures. AIAA Journal, 31, 1280-1286. https://doi.org/10.2514/3.11764

[10] Ray, M.C., Rao, M.K. and Samanta, B. (1992) Exact Analysis of Coupled Electroelastic Behavior of a Piezoelectric Plate under Cylindrical Bending. Computers and Structures, 45, 667-677. https://doi.org/10.1016/0045-7949(92)90485-I

[11] Ray, M.C., Rao, K.M. and Samanta, B. (1993) Exact Solution for Static Analysis of Intelligent Structures under Cylindrical Bending. Computers and Structures, 47, 1031-1042. https://doi.org/10.1016/0045-7949(93)90307-Y

[12] Ray, M.C. (1998) Optimal Control of Laminated Plates with Piezoelectric Sensor and Actuator Layers. AIAA Journal, 36, 2204-2208. https://doi.org/10.2514/2.345

[13] Ray, M.C. (1998) Closed form Solution for Optimal Control of Laminated Plate. Computers and Structures, 69, 283-290.

https://doi.org/10.1016/S0045-7949(97)00093-X 
[14] Ray, M.C., Bhattacharyya, R. and Samantha, B. (1998) Exact Solution for Dynamic Analysis of Composite Structures with Distributed Piezoelectric Sensors and Actuators. Computers and Structures, 66, 737-743.

https://doi.org/10.1016/S0045-7949(97)00126-0

[15] Ray, M.C., Bhattacharyya, R. and Samantha, B. (1996) Finite Element Model for Active Control of Intelligent Structures. AIAA Journal, 34, 1885-1893.

https://doi.org/10.2514/3.13322

[16] Krommer, M. (2001) On the Correction of the Bernuoulli-Euler Beam Theory for Smart Piezoelectric Beams. Smart Materials and Structures, 10, 668-680. https://doi.org/10.1088/0964-1726/10/4/310

[17] Rao, S.S. and Sunar, M. (1994) Piezoelectricity and Its Use in Disturbance Sensing And Control of Flexible Structures: A Survey. Applied Mechanics Reviews, 47, 113-123. https://doi.org/10.1115/1.3111074

[18] Moita, J.S.M., Coreia, I.F.P., Soares, C.M.M. and Soares, C.A.M. (2004) Active Control of Adaptive Laminated Structures with Bonded Piezoelectric Sensors and Actuators. Computers and Structures, 82, 1349-1358. https://doi.org/10.1016/j.compstruc.2004.03.030

[19] Gabbert, U., Tamara, N.T. and Kppel, H. (2002) Modeling, Control and Simulation of Piezoelectric Smart Structures Using Finite Element Method and Optimal LQ Control. Facta Universitatis Series. Mechanics, Automatic Control and Robotics, 12 , 417-430.

[20] Lim, Y.-H., Gopinathan, S.V., Vardan, V.V. and Vardan, V.K. (1999) Finite Element Simulation of Smart Structures Using an Optimal Output Feedback Controller for Vibration and Noise Control. International Journal of Smart Materials and Structures, 8, 324-337. https://doi.org/10.1088/0964-1726/8/3/305

[21] Aldraihem, O.J., Wetherhold, T. and Sigh, T. (1997) Distributed Control of Laminated Beams: Timoshenko vs. Euler-Bernoulli Theory. Journal of Intelligent Materials Systems and Structures, 8, 149-157. https://doi.org/10.1177/1045389X9700800205

[22] Abramovich, H. (1998) Deflection Control of Laminated Composite Beam with Piezoceramic Layers-Closed form Solutions. Composite Structures, 43, 217-231. https://doi.org/10.1016/S0263-8223(98)00104-4

[23] Chandrashekara, K. and Vardarajan, S. (1997) Adaptive Shape Control of Composite Beams with Piezoelectric Actuators. Intelligent Materials Systems and Structures, 8, 112-124. https://doi.org/10.1177/1045389X9700800202

[24] Sun, C.T. and Zhang, X.D. (1995) Use of Thickness Shear Mode in Adaptive Sandwich Structures. Smart Materials and Structures, 3, 202-206.

https://doi.org/10.1088/0964-1726/4/3/007

[25] Aldraihem, O.J. and Khedir Ahmed, A. (2000) Smart Beams with Extension and Thickness Shear Piezoelectric Actuators. Journal of Smart Materials and Structures, 9, 1-9. https://doi.org/10.1088/0964-1726/9/1/301

[26] Zhang, X.D. and Sun, C.T. (1996) Formulation of an Adaptive Sandwich Beam. Smart Materials and Structures, 5, 814-823.

https://doi.org/10.1088/0964-1726/5/6/012

[27] Donthireddy, P. and Chandrashekhara, K. (1996) Modeling and Shape Control of Composite Beam with Embedded Piezoelectric Actuators. System and Control Lectures, 35, 237-244. https://doi.org/10.1016/0263-8223(96)00041-4

[28] Rathi, V. and Khan, A.H. (2012) Vibration Attenuation and Shape Control of Sur- 
face Mounted, Embedded Smart Beam. Latin American Journal of Solids and Structures, 9, 401-424. https://doi.org/10.1590/S1679-78252012000300006

[29] Chammas, A.B. and Leondes, C.T. (1979) Pole Placement by Piecewise Constant Output Feedback. International Journal of Control, 29, 31-38. https://doi.org/10.1080/00207177908922677

[30] Chammas, A.B. and Leondes, C.T. (1978) On the Design of LTI Systems by Periodic Output Feedback Part-II, Output Feedback Controllability. International Journal of Control, 27, 895-903. https://doi.org/10.1080/00207177808922420

[31] Werner, H. and Furuta, K. (1995) Simultaneous Stabilization Based on Output Measurements. Kybernetika, 31, 395-411.

[32] Werner, H. (1998) Multimodal Robust Control by Fast Output Sampling-An LMI Approach. Automatica, 34, 1625-1630.

https://doi.org/10.1016/S0005-1098(98)80018-6

[33] Janardhanan, S., Bandyopahyay, B. and Manjunath, T.C. (2004) Fast Output Sampling Based Output Feedback Sliding Mode Control Law for Uncertain Systems. Proceedings of the Third International Conference on System Identification and Control Problems, Moscow, 28-30 January 2004, Paper No. 23010, 1300-1312.

[34] Bandyopahyay, B., Thakkar, V., Saaj, C.M. and Janardhanan, S. (2004) Algorithm for Computing Sliding Mode Control and Switching Surfaces from Output Samples. Proceedings of the $8^{\text {th }}$ IEEE VSS Workshop, Spain, No. 4.

[35] Bartoszewicz, A. (1998) Discrete-Time Quasi-Sliding-Mode Control Strategies. IEEE Transactions on Industrial Electronics, 45, 633-637. https://doi.org/10.1109/41.704892

[36] Werner, H. (1996) Robust Control of a Laboratory Flight Simulator by Non-Dynamic Multirate Output Feedback. Proceeding IEEE Conference on Decision and Control, 2, 1575-1580. https://doi.org/10.1109/CDC.1996.572749

[37] Gao, W., Wang, Y. and Homaifa, A. (1995) Discrete-Time Variable Structure Control Systems. IEEE Transactions on Industrial Electronics, 42, 117-122. https://doi.org/10.1109/41.370376

[38] Swaminadham, M., et al. (1989) Non-Contact Blade Deflection Measurement System for Rotating Bladed Disks. Proceedings of $35^{\text {th }}$ International Instrumentation Symposium, Instrument Society of America, 455-468.

[39] Rathi, V., Khan, A.H. and Khan, A.A. (2012) Active Vibration Control of Smart Beams with Embedded Actuators and Sensors. Proceedings of $3^{\text {rd }}$ Asian Conference on Mechanics of Functional Materials and Structures, Delhi, 127-130.

[40] Rathi, V., Khan, A.A. and Maheshwari, S. (2013) Active Vibration Control of Embedded Beam POF vs. FOS. Proceedings of National Conference on Recent Trends in Mechanical and Production Engineering, 305-319.

[41] Rathi, V. and Khan, A.A. (2016) Vibration Obliteration of Smart Cantilever Beam with SISO DTSMC. Proceedings of National Conference on Mechanical Engineering Ideas, Innovations and Initiatives, 150-155. 


\section{List of Acronyms/Abbreviations}

AVC: Active Vibration Control

CT: Continuous Time

DT: Discrete Time

DQSMC: Discrete Quasi Sliding Mode Control

DSMC: Discrete Sliding Mode Control

DTSMC: Discrete Time Sliding Mode Control

EBT: Euler-Bernoulli Beam Theory

FE: Finite Element

FOS: Fast Output Sampling

FSDT: First Order Shear Deformation Theory

LTI: Linear Time Invariant

MATLAB: MATrix LABoratory

MROF: Multi Rate Output Feedback

MIMO: Multiple Input Multiple Output

POF: Periodic Output Feedback

PZT: Lead Zirconate Titanate

SISO: Single Input Single Output

SS: State Space

TBT: Timoshenko Beam Theory 


\section{Appendix}

1) The Material Constants are

$$
Q_{11}=\frac{E_{11}}{1-v_{12} v_{21}}, Q_{22}=\frac{E_{22}}{1-v_{12} v_{21}}, Q_{12}=\frac{v_{12} E_{11}}{1-v_{12} v_{21}}, \frac{v_{12}}{E_{11}}=\frac{v_{21}}{E_{22}}, Q_{66}=G_{12}
$$

2) The Shape Functions are

$$
\begin{aligned}
& N_{1}=1-\frac{x}{l}, \quad N_{2}=\frac{6 \mu}{\Omega} x-\frac{6 \mu}{l \Omega} x^{2}, \quad N_{3}=-\frac{6 \mu}{\Omega} x+\frac{6 \mu}{l \Omega} x^{2}, \quad N_{4}=\frac{x}{l}, \\
& N_{5}=\frac{3 \mu l}{\Omega} x+\frac{3 \mu}{l \Omega} x^{2}, \quad N_{6}=-\frac{3 \mu l}{\Omega} x+\frac{3 \mu}{l \Omega} x^{2}, \quad N_{7}=1-\frac{12 \lambda}{l \Omega} x+\frac{3}{\Omega} x^{2}-\frac{2}{l \Omega} x^{2}, \\
& N_{8}=\left(\frac{6 \lambda}{\Omega}-1\right) x-\frac{x^{2}}{2 l}-\frac{3}{2} \frac{l}{\Omega} x^{2}+\frac{1}{\Omega} x^{3}, \quad N_{9}=\frac{12 \lambda}{l \Omega} x-\frac{3}{\Omega} x^{2}+\frac{2}{l \Omega} x^{3}, \\
& N_{10}=\frac{6 \lambda}{\Omega} x-\frac{x^{2}}{2 l}-\frac{3}{2} \frac{l}{\Omega} x^{2}+\frac{1}{\Omega} x^{3}, \quad N_{11}=-\frac{6}{\Omega} x+\frac{6}{l \Omega} x^{2}, \quad N_{12}=1-\frac{x}{l}+\frac{3 l}{\Omega} x-\frac{3}{\Omega} x^{2}, \\
& N_{13}=-\frac{6}{\Omega} x-\frac{6}{l \Omega} x^{2}, \quad N_{14}=\frac{x}{l}+\frac{3 l}{\Omega} x-\frac{3}{\Omega} x^{2}
\end{aligned}
$$

3) Mass Matrix Coefficients for Embedded Beam Element $\left(m_{i j}\right)$ :

$$
\begin{aligned}
m_{11}= & \frac{I_{1} l}{3}, \quad m_{12}=\frac{\mu I_{1} l^{2}}{2 \Omega}=m_{21}, \quad m_{13}=-\frac{\mu I_{1} l^{3}}{4 \Omega}=m_{31}, \quad m_{14}=\frac{I_{1} l}{6}=m_{41}, \\
m_{15}= & -\frac{\mu I_{1} l^{2}}{2 \Omega}=m_{51}, \quad m_{16}=-\frac{\mu I_{1} l^{3}}{4 \Omega}=m_{61}, \\
m_{22}= & \frac{l}{35 \Omega^{2}}\left[13 I_{3} l^{4}+35 I_{2} l^{3}+42\left\{I_{1}\left(1+\mu^{2}\right)-7 I_{3} \lambda\right\} l^{2}-420 I_{2} \lambda l+1680 I_{3} \lambda^{2}\right], \\
m_{23}= & \frac{-l}{210 \Omega^{2}}\left[1 I_{3} l^{5}+21\left\{I_{1}\left(1+6 \mu^{2}\right)-11 I_{3} \lambda\right\} l^{3}+840 I_{2} \lambda l^{2} \quad m_{24}=\frac{\mu l^{2} I_{1}}{\Omega}=m_{42},\right. \\
& \left.+1260 \lambda\left(I_{3} \lambda+I_{1}\right) l-10080 I_{2} \lambda^{2}\right]=m_{32}, \\
m_{44}= & \frac{I_{1} l}{3}, \quad m_{25}=\frac{3 l}{70 \Omega^{2}}\left[3 I_{2} l^{4}-28\left\{I_{1}\left(1+\mu^{2}\right)+3 I_{3} \lambda\right\} l^{2}+560 I_{3} \lambda^{2}\right]=m_{52}, \\
m_{26}= & \frac{l}{420 \Omega^{2}}\left[13 I_{3} l^{5}-42\left\{I_{1}\left(6 \mu^{2}+1\right)+9 I_{3} \lambda\right\} l^{3}-840 I_{2} \lambda l^{2}+2520 \lambda\left(I_{3} \lambda-I_{1}\right) l+10080 I_{2} \lambda^{2}\right]=m_{62} ; \\
m_{33}= & \frac{l}{210 \Omega^{2}}\left[2 I_{1} l^{6}+7\left\{I_{1}\left(9 \mu^{2}+4\right)-6 I_{3} \lambda\right\} l^{4}+210 I_{2} \lambda l^{3}+84 \lambda\left(3 I_{3} \lambda-5 I_{1}\right) l^{2}-2520 I_{2} \lambda^{2} l+10080 I_{1} \lambda^{2}\right] ; \\
m_{34}= & \frac{\mu I_{1} l^{3}}{4 \Omega}=m_{43} ; \\
m_{33}= & \frac{l}{210 \Omega^{2}}\left[2 I_{1} l^{6}+7\left\{I_{1}\left(9 \mu^{2}+4\right)-6 I_{3} \lambda\right\} l^{4}+210 I_{2} \lambda l^{3}+84 \lambda\left(3 I_{3} \lambda-5 I_{1}\right) l^{2}-2520 I_{2} \lambda^{2} l+10080 I_{1} \lambda^{2}\right] ; \\
m_{36}= & -\frac{l}{420 \Omega}\left[3 I_{3} l^{6}+14\left\{I_{1}\left(\lambda^{2}-9 \mu^{2}\right)-6 I_{3} \lambda\right\} l^{4}+168\left(3 I_{3} \lambda-5 I_{1}\right) l^{2}-10080 I_{1} \lambda^{2}\right]=m_{63} ; \\
m_{45}= & \frac{\mu I_{1} l^{2}}{2 \Omega}=m_{54} ; \quad m_{46}=\frac{\mu I_{1} l^{3}}{4 \Omega}=m_{64} ; \\
m_{55}= & \frac{l}{35 \Omega^{2}}\left[13 I_{3} l^{4}-35 I_{2} l^{3}+42\left\{I_{1}\left(\mu^{2}+1\right)-7 \mu I_{3}\right\} l^{2}+420 I_{2} \lambda l+1680 I_{3} \lambda^{2}\right] ; \\
m_{56}= & \frac{l}{210 \Omega^{2}}\left[11 I_{3} l^{5}+21\left\{I_{1}\left(1+6 \mu^{2}\right)-11 I_{3} \lambda\right\} l^{3}-840 I_{2} \lambda l^{2}+1260 \lambda\left(I_{1}+I_{3} \lambda\right) l+10080 I_{2} \lambda^{2}\right]=m_{65} ; \\
m_{66}= & \frac{l}{210 \Omega^{2}}\left[2 I_{3} l^{6}+7\left\{I_{1}\left(9 \mu^{2}+4\right)-6 I_{2} \lambda\right\} l^{4}-210 I_{2} \lambda l^{3}+84\left(3 I_{3}-5 I_{1}\right) l^{2}+2520 I_{2} \lambda^{2} l+10080 I_{2} \lambda^{2}\right]
\end{aligned}
$$


4) Stiffness Matrix Coefficients for Embedded Beam $\left(k_{i j}\right)$ :

$$
\begin{aligned}
& k_{11}=\frac{A A_{11}}{l} ; \quad k_{12}=\frac{A B_{11}}{l}=k_{21} ; \quad k_{13}=0=k_{31} ; \quad k_{14}=-\frac{A A_{11}}{l}=k_{41} ; \\
& k_{15}=-\frac{A B_{11}}{l}=k_{51} ; k_{16}=0=k_{61} ; \quad k_{24}=-\frac{A B_{11}}{l}=k_{42} ; \quad k_{34}=0=k_{43} ; \\
& k_{22}=\frac{-A l}{10 \Omega^{2}}\left\{D_{11} I^{3}-10 B_{11} \mu l^{2}+60\left(A_{55}+A_{11} \lambda^{2}\right) l+120 B_{11} \mu \lambda\right\} \\
& k_{23}=\frac{6 A}{5 l \Omega^{2}}\left\{D_{11} l^{4}+10\left(A_{55}+A_{11} \mu^{2}-2 D_{11} \lambda\right) l^{2}+120 D_{11} \lambda^{2}\right\}=k_{32} ; \\
& k_{25}=-\frac{6 A}{5 l \Omega^{2}}\left\{D_{11} l^{4}+10\left(A_{55}+A_{11} \mu^{2}-2 D_{11} \lambda\right) l^{2}+120 D_{11} \lambda^{2}\right\}=k_{52} ; \\
& k_{26}=\frac{A l}{10 \Omega^{2}}\left\{-D_{11} l^{3}-10 B_{11} \mu l^{2}-60\left(A_{55}+A_{11} \lambda^{2}\right) l+120 B_{11} \mu \lambda\right\}=k_{62} ; \\
& k_{33}=\frac{A}{15 l \Omega^{2}}\left\{2 D_{11} I^{6}-15 B_{11} \mu l^{5}+15\left(3 A_{11} \mu^{2}-2 D_{11} \lambda+4 A_{55}\right) l^{4}+180 B_{11} \mu \lambda l^{3}+180\left(D_{11} \lambda^{2}-2 A_{55}\right) l^{2}+2160 A_{55} \lambda^{2}\right\} ; \\
& k_{35}=\frac{A l}{10 \Omega^{2}}\left\{D_{11} 1^{3}-10 B_{11} \mu l^{2}+60\left(A_{55}+A_{11} \lambda^{2}\right) l+120 B_{11} \mu \lambda\right\}=k_{53} ; \\
& k_{36}=-\frac{A}{30 l \Omega^{2}}\left\{D_{11} l^{6}-30\left(2 A_{55}-3 A_{11} \mu^{2}-2 D_{11} \lambda\right) l^{4}+360 \lambda\left(D_{11} \lambda-2 A_{55}\right) l^{2}+4320 A_{55} \lambda^{2}\right\}=k_{63} ; \\
& k_{44}=\frac{A A_{11}}{l} ; \quad k_{45}=\frac{A B_{11}}{l}=k_{54} ; \quad k_{46}=0=k_{64} ; \\
& k_{55}=\frac{6 A}{5 l \Omega^{2}}\left\{D_{11} I^{4}+10\left(A_{55}+A_{11} \mu^{2}-2 D_{11} \lambda\right) l^{2}+120 D_{11} \lambda^{2}\right\} ; \\
& k_{56}=-\frac{A l}{10 \Omega^{2}}\left\{-D_{11} l^{3}-10 B_{11} \mu l^{2}-60\left(A_{55}+A_{11} \lambda^{2}\right) l+120 B_{11} \mu \lambda\right\}=k_{65} ; \\
& k_{66}=\frac{A}{15 l \Omega^{2}}\left\{2 D_{11} l^{6}+15 B_{11} \mu l^{5}+15\left(3 A_{11} \mu^{2}-2 D_{11} \lambda+4 A_{55}\right) l^{4}-180 B_{11} \mu \lambda l^{3}+180\left(D_{11} \lambda^{2}-2 A_{55}\right) l^{2}+2160 A_{55} \lambda^{2}\right\} ;
\end{aligned}
$$

Here, $\Omega=\left(12 \lambda-l^{2}\right), \mu=\frac{B_{11}}{A_{11}}$ and $\lambda=\frac{D_{11}}{A_{55}}\left(\frac{\mu B_{11}}{D_{11}}-1\right)$ 